\title{
NEUTRINO TRANSPORT IN TYPE II SUPERNOVAE : BOLTZMANN SOLVER VS MONTE CARLO METHOD
}

\author{
SHOICHI YAMADA AND HANS-THOMAS JANKA \\ Max-Planck-Institut für Astrophysik \\ Karl - Schwarzschild - Str. 1, D-85740 Garching, Germany \\ AND \\ HIDEYUKI SUZUKI \\ National Laboratory for High Energy Physics (KEK) \\ Oho, Tsukuba, Ibaraki 305, Japan
}

In this paper we discuss the results of extensive comparison of the Boltzmann solver recently developed by us and the Monte Carlo method coded by Janka. The aim of our project is to improve the treatment of the neutrino transfer and to study the neutrino reactions systematically, since the numerical treatment of neutrino transfer and neutrino reaction rates in the hot dense medium are two of the greatest uncertainties in the supernova simulations.

We assume spherical symmetry of the system. The fully general relativistic Boltzmann equation for the Misner-Sharp metric is finite differenced with respect to each coordinate, time $t$, baryon mass $m$, directional cosine of neutrino 3-momentum $\mu$ and neutrino energy $\varepsilon_{\nu}$, and solve the resulting coupled equations. In the Monte Carlo simulations, on the other hand, $\sim 500,000$ particles are typically used. We used Wilson's realistic models for three different times after core bounce, and calculated the neutrino transport for this static backgrounds.

In the typical calculations, the Boltzmann solver used 105 spatial grids, 6 angular meshes in 180 degrees, 12 energy bins covering $0 \sim 110 \mathrm{MeV}$. However, these numbers are varied systematically to see the effect of the resolution.

As shown in Figure 1, the number density and the number flux as well as the energy spectrum of neutrinos are quite well reproduced by the Boltzmann solver. Figure $2 \mathrm{a}$, on the other hand, clearly shows the limitation of the Boltzmann solver. The energy integrated flux factor and Eddington fac- 

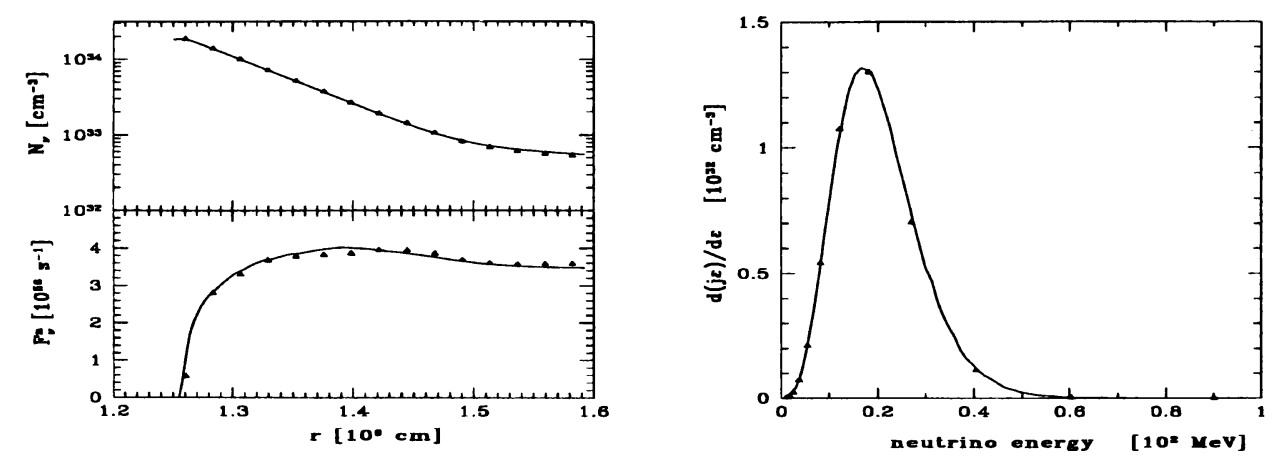

Figure 1. a. The number density (upper panel) and the number flux (lower panel) of the electron-type neutrino at $t=3.32 \mathrm{sec}$. Triangles are for Monte Carlo results. $b$. The electron-type anti-neutrino energy spectrum at $t=3.32 \mathrm{sec}$. Triangles are for Boltzmann results.
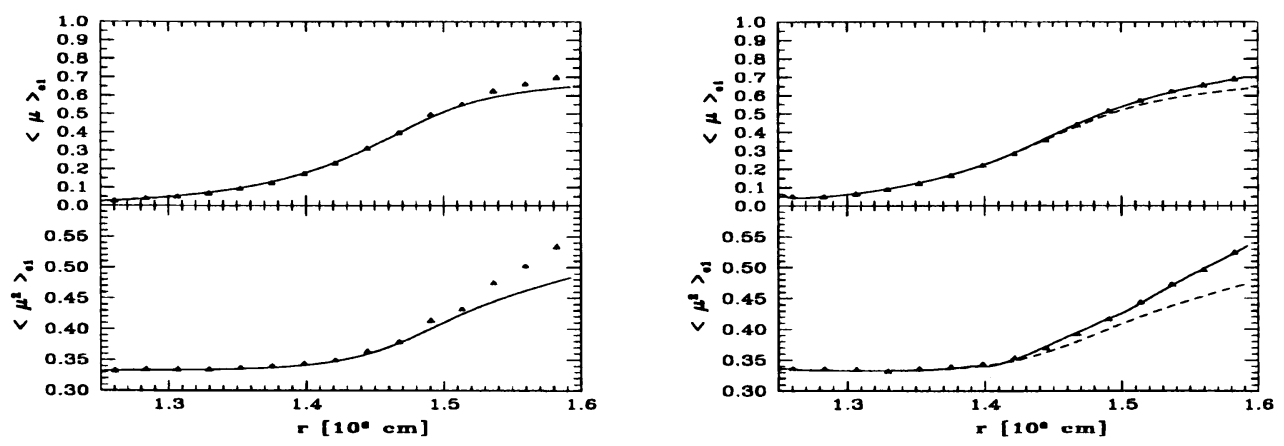

Figure 2. The flux factor and Eddington factor for the muon-type neutrino with (a) and without (b) a variable angular mesh method.

tor are considerably underestimated by the Boltzmann solver in the outer region where the neutrino angular distribution is forward peaked. This is entirely due to the poor angular resolution of the Boltzmann solver. This is a serious drawback of the Boltzmann solver in studying the neutrino heating mechanism behind shock where the very forward peaked angular distribution is expected. To improve the angular resolution without increasing the mesh number we implemented the variable angular mesh method in which the angular mesh is collected more in the forward direction depending on the time and position. As shown in Figure $2 b$ both the flux factor and Eddington factor are improved remarkably. 